\title{
The electrochemical detection of 4-chlorophenol at gold electrodes modified with different phthalocyanines
}

\author{
Karl Peeters, Karolien De Wael ${ }^{1}$, David Bogaert, Annemie Adriaens* \\ Department of Analytical Chemistry, Ghent University, Krijgslaan 281-S12, B-9000 Ghent, Belgium \\ Received 21 March 2007; received in revised form 6 July 2007; accepted 7 July 2007 \\ Available online 13 July 2007
}

\begin{abstract}
Because of their toxicity to humans and their tendency to bioaccumulate in the food chain, the detection of chlorophenols has been investigated intensively. This paper compares the oxidation of 4-chlorophenol on a bare gold electrode to its detection on gold electrodes electrochemically modified with either cobalt(II) tetrasulphonated phthalocyanine (CoTSPc) or 3,4',4", $4^{\prime \prime \prime}$-copper(II) tetrasulphonated phthalocyanine (CuTSPc). Results show that the electrochemical behaviour of 4-chlorophenol depends on the molecular structure of the phthalocyanine thin film layers on the gold surface. In addition, the influence of the coordinating properties of the phthalocyanines on the sensitivity of the measurement and the fouling of the electrode material is discussed.
\end{abstract}

(C) 2007 Elsevier B.V. All rights reserved.

Keywords: Detection; Chlorophenol; Gold electrode; Phthalocyanine; Modification

\section{Introduction}

Chlorophenols have been recognized as environmental pollutants since the 1960s because of their toxicity to humans and most aquatic organisms and their tendency to bioaccumulate in the food chain [1]. They are known to be widespread in industrial wastes (e.g. pulp and paper mills, petrochemical refineries and coke plants [2]) and are used in many industrial processes such as the manufacture of plastics, dyes and pesticides [3]. In addition, these compounds are applied as wood preservatives in agricultural and domestic applications and in additives to inhibit microbial growth in a wide array of products, such as adhesives, oils, textiles and pharmaceutical products [2].

In the past different chromatographic and spectroscopic techniques have been tested and used for the detection of chlorophenols in wastewater [3-5]. These methods, however, can be quite time consuming. Moreover, the fact that samples need to be taken from the site into the laboratory potentially implies the possibilities for sample contamination, alteration or fraud. This makes that there is a need for sensitive, repro-

\footnotetext{
* Corresponding author. Tel.: +329264 4826; fax: +329264 4960 .

E-mail address: annemie.adriaens@ugent.be (A. Adriaens).

${ }^{1}$ Postdoctoral fellow of the Research Foundation, Flanders, Belgium.
}

ducible, stable, easy-to-use and low-cost analytical methods for monitoring chlorophenols in water.

In more recent years the electrochemical detection of chlorophenols and the development of an electrochemical sensor for these compounds have been investigated intensively together with their degradation and adsorption [6-8]. Different bare electrode materials such as gold, platinum, glassy carbon and boron doped diamond electrodes have been tested [4,9-13] as well as modified electrodes with different kinds of catalysts $[14,15]$. Especially the use of transition metallo phthalocyanines as electrocatalysts has lately attracted a wide research interest [14-18]. Soluble sulphonated phthalocyanines have shown to be able to form layers on different types of supporting material, including electrodes [19]. The immobilization of these compounds can lead to modified electrodes with improved kinetic properties. The major characteristics of phthalocyanines are their high thermal and chemical stability, coupled with their extensive redox chemistry and their tendency to form aggregates [20-22].

In previous studies, the electrodeposition of cobalt(II) tetrasulphonated phthalocyanine (CoTSPc) or $3,4^{\prime}, 4^{\prime \prime}, 4^{\prime \prime \prime}$-copper(II) tetrasulphonated phthalocyanine (CuTSPc) on gold electrodes in a pH 12 buffer solution has been described [19,23,24]. In addition, the morphology and heterogeneity of the adsorbed CoTSPc or CuTSPc thin layers were studied with synchrotron radiation $\mathrm{X}$-ray fluorescence spectroscopy. The latter allowed the authors 
to develop a theory about the layer formation and build-up of CoTSPc as well as CuTSPc on gold electrodes [25-27].

In this work, the oxidation of 4-chlorophenol (4-Cp) on bare gold electrodes is compared to its detection on gold electrodes electrochemically modified with CoTSPc or CuTSPc. Results show that the electrochemical behaviour of 4-chlorophenol depends on the molecular structure of the phthalocyanine thin layers on the gold surface. In addition, the influence of the coordinating properties of the phthalocyanines on the sensitivity of the measurement and the fouling of the electrode material is discussed.

\section{Experimental}

The immobilization of a phthalocyanine (CoTSPc or CuT$\mathrm{SPc}$ ) on a gold electrode was performed in a three-electrode cell with a saturated calomel reference electrode (SCE) with two compartments (Radiometer Analytical, France) and a carbon counter electrode. The working electrodes were gold disc electrodes with a diameter of $1.6 \mathrm{~mm}$ (BASI, UK), which were pretreated by mechanical and electrochemical polishing according to the following procedure. Before its first use, the electrode surface was briefly scoured by a silicon carbide emery paper of 1200 grit to obtain a fresh surface. To smoothen the resulting relatively rough surface it was further subjected to sequential polishing on a polishing cloth covered with alumina powder of $1,0.3$ and $0.05 \mu \mathrm{m}$ particle size (Buehler, USA) for respectively 5, 10 and $20 \mathrm{~min}$. To remove any adherent $\mathrm{Al}_{2} \mathrm{O}_{3}$ particles the electrode surface was rinsed thoroughly with doubly deionised water and cleaned in an ultrasonic bath containing deionised water (Branson 3210, USA) for $2 \mathrm{~min}$. Finally, the electrode was pretreated electrochemically by scanning it in a $0.1 \mathrm{~mol} \mathrm{~L}^{-1} \mathrm{Na}_{2} \mathrm{HPO}_{4} / \mathrm{NaOH}$ buffer solution ( $\mathrm{pH}$ 12) between -1.2 and $0.6 \mathrm{~V}$ versus SCE until five subsequent scans were identical.

The modification of the electrodes was done by recording successive cyclic voltammetric scans in a potential window from -1.2 to $0.6 \mathrm{~V}$ versus $\mathrm{SCE}\left(50 \mathrm{mV} \mathrm{s}^{-1}\right)$ during a defined number of scans in a $\mathrm{pH} 12$ buffer solution containing either CoTSPc or CuTSPc. For a more detailed description of the electrochemical behaviour of CoTSPc or CuTSPc on a gold electrode, the reader is referred to the literatures $[19,23]$. Important for this study is that the modification was performed prior, at and after the breaking point (i.e. the scan at which there is a maximum adsorption) for the modification with CoTSPc and after 10, 60 and 100 scans for the modification with CuTSPc (no breaking point was observed for CuTSPc).

After the modification step, the modified electrode was scanned during 20 voltammetric scans in a blank pH 12 buffer solution followed by adding different concentrations of 4-Cp. For each concentration, a freshly made modified electrode was used.

A PGSTAT20 potentiostat controlled by GPES 4.9005 software package running (ECO Chemie, The Netherlands) was used to record the voltammetric curves. Before the start of each measurement, the $\mathrm{pH}$ of the solution was measured using an
Orion Benchtop pH-meter model 420A (Thermo Fisher Scientific, USA).

The CoTSPc sodium salts, with the four sulphonated groups randomly placed over the phthalocyanine structure, were purchased from Rhodes University of Grahamstown (Eastern Cape, South Africa). The CuTSPc sodium salts were purchased from Sigma-Aldrich (USA). In case of the latter, the four sulphonated groups can be found on fixed positions, more specifically on the $3,4^{\prime}, 4^{\prime \prime}$ and $4^{\prime \prime \prime}$ spot of a phthalocyanine ring (CuTSPc). The buffer solution of $\mathrm{pH} 12\left(\mathrm{Na}_{2} \mathrm{HPO}_{4} / \mathrm{NaOH}\right)$ and the 4$\mathrm{Cp}$ were purchased from VWR-International (Belgium). Before each experiment, pure nitrogen was bubbled through the cell solution for $20 \mathrm{~min}$.

The oxidation peak current $(I)$ of $4-\mathrm{Cp}$ of the first voltammetric scan was plotted as a function of the concentration $\left(C_{\mathrm{Cp}}\right)$ to obtain calibration curves in a concentration range from 0.05 to $1 \mathrm{mmol} \mathrm{L}^{-1}$. Linear regression by the least-squares method was applied using the model: $I=b_{0}+b_{1} C_{\mathrm{Cp}}+e$, where $e$ is the residual. The uncertainty of these values $\left(s_{b_{0}}\right.$ and $\left.s_{b_{1}}\right)$ was calculated next to the pure (experimental) error $\left(s_{\mathrm{y}}\right)$. The latter were statistically compared on the $95 \%$ confidence level using the $F$-test for the precision. The calibration curves have been statistically compared with each other on the $95 \%$ confidence level with the two-tailed $t$-test using the Bonferroni adjustment. This adjustment was used since more than two means were compared which makes that the same mean is used several times and consequently the $t$-tests are not independent of each other. As a result, when all population means are equal, the probability that at least one comparison will be found to be significant different increases [28].

\section{Results}

\subsection{Oxidation potential of 4-Cp}

Fig. 1 shows the first cyclic voltammetric scan of $1.0 \mathrm{mmol} \mathrm{L}^{-1}$ 4-Cp on a bare gold electrode (a, curve 1), on a gold electrode modified with CoTSPc until the breaking point (b, curve 1) and on a gold electrode modified with CuTSPc during 60 subsequent voltammetric scans (c, curve 1). Also the current potential curves recorded at the three electrodes (respectively a, b and c, curve 2) in a 4-Cp free $\mathrm{pH} 12$ buffer solution are shown. Next to the peaks related to the adsorbed phthalocyanine, the oxidation of gold with the formation of gold oxides (around $0.4 \mathrm{~V}$ versus SCE) and the reduction of the formed gold oxides with regeneration of the original gold surface (around $0.15 \mathrm{~V}$ versus SCE) can be seen in curves 2 . Curves 1 show that $4-C p$ can be oxidized at a bare gold electrode, as well as at modified electrodes. The oxidation process of 4-Cp occurs at around $0.5 \mathrm{~V}$ versus SCE, the same potential at which the gold oxidation takes place. It has been postulated that the oxidation of chlorophenol begins with the interaction of the chlorophenol molecule with an adsorbed $\mathrm{OH}$, which has been formed by electro-oxidation of an $\mathrm{Au}-\mathrm{H}_{2} \mathrm{O}$ site. In this way, a phenoxy radical is formed which on its turn can start a polymerization reaction. Stabilization of the radical occurs by the delocalization of the radical centre over the aromatic ring $[9,12]$. 

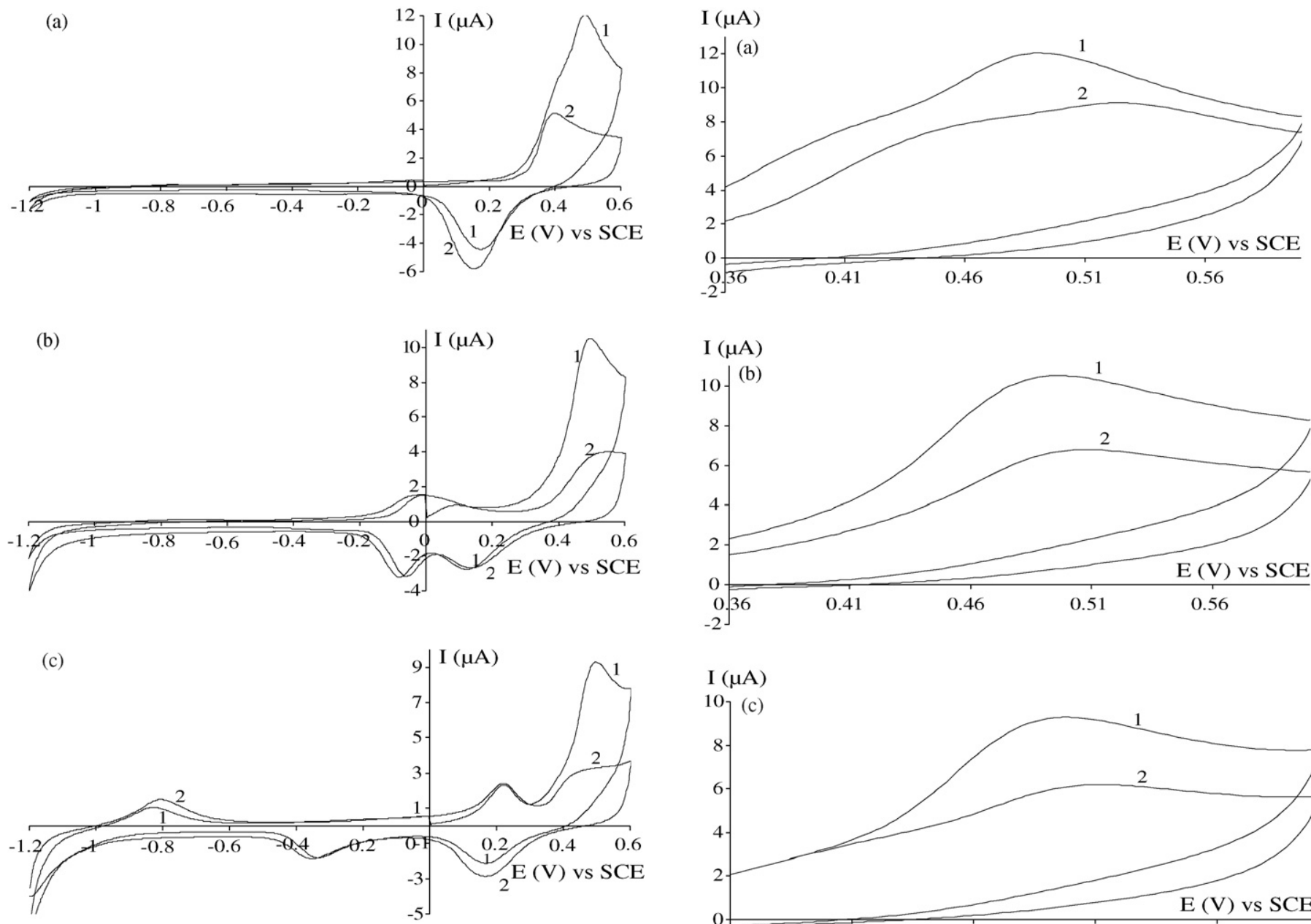

Fig. 1. Current potential curves recorded with a scan rate of $50 \mathrm{mV} \mathrm{s}^{-1}$ between 0.6 and $-1.2 \mathrm{~V}$ vs. SCE in a $1.0 \mathrm{mmol} \mathrm{L}^{-1} 4-\mathrm{Cp} \mathrm{pH} 12$ buffer solution at a bare gold electrode (a), at a gold electrode modified during 60 scans in $4 \mathrm{mmol} \mathrm{L}^{-1}$ CuTSPc (b) and at a gold electrode modified in $6 \mathrm{mmol} \mathrm{L}^{-1}$ CoTSPc till the breaking point (c) at $298 \mathrm{~K}$ (curves 1). Also the current potential curves recorded under the same conditions of the same electrodes in a 4-Cp free $\mathrm{pH} 12$ buffer solution are shown (curves 2).

The oxidation of 1.0 and $4.0 \mathrm{mmol} \mathrm{L}^{-1} 4-\mathrm{Cp}$ at bare gold (a) and at modified electrodes (b for CuTSPc and c for CoT$\mathrm{SPc}$ ) is shown in more detail in Fig. 2. The peak potential of the oxidation of 4-Cp at a bare gold electrode shifts over $30 \mathrm{mV}$ to higher potentials when the 4-Cp concentration is changed from 1.0 to $0.4 \mathrm{mmol} \mathrm{L}^{-1}$. Moreover, it is noticed that the 4-Cp oxidation peak interferes with the gold oxidation peak at circa $0.4 \mathrm{~V}$ versus SCE. However, the peak potentials of the oxidation of 4-Cp at modified electrodes show only a minor change when the $4-\mathrm{Cp}$ concentration is changed.

\subsection{Fouling of the electrode materials}

Fig. 1 gives a first indication of the fouling of the electrode in the presence of 4-Cp. The decrease of the reduction peak at $0.15 \mathrm{~V}$ versus SCE (reduction of gold oxides) in the presence of 4-Cp indicates that the electrode is poisoned. A fouling of

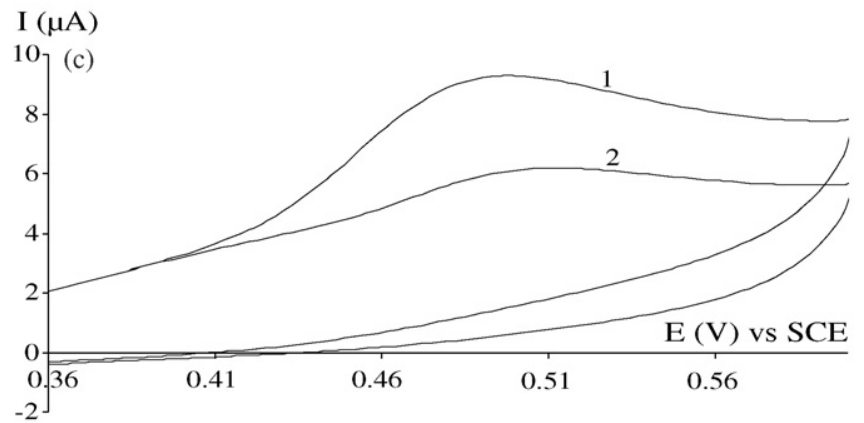

Fig. 2. Current potential curves recorded with a scan rate of $50 \mathrm{mV} \mathrm{s}^{-1}$ between 0.6 and $-1.2 \mathrm{~V}$ vs. SCE in a $\mathrm{pH} 12$ buffer solution containing $1.0 \mathrm{mmol} \mathrm{L}^{-1}$ (1) or $0.4 \mathrm{mmol} \mathrm{L}^{-1}$ (2) 4-Cp at a bare gold electrode (a), at a gold electrode modified during 60 scans in $4 \mathrm{mmol} \mathrm{L}^{-1} \mathrm{CuTSPc}$ (b) and at a gold electrode modified with CoTSPc till the breaking point (c).

the electrode results in a smaller amount of free gold and thus a decrease of the corresponding redox peaks.

A second indication is given in Fig. 3 which shows a detail of the oxidation of $0.6 \mathrm{mmol} \mathrm{L}^{-1} 4-\mathrm{Cp}$ at a gold electrode modified with CoTSPc till the breaking point (a-e) and with CuTSPc during 60 voltammetric scans $(\mathrm{f}-\mathrm{j})$ as a function of time (voltammetric scan number). It is seen that the current decreases with growing voltammetric scan number indicating the fouling of the electrode and diminishing the number of active sites.

According to the literature this poisoning phenomenon can be explained by the formation of phenoxy radicals which can react through two pathways [9,29-31]. Gattrell and Kirk [29-31] describe the chlorophenol oxidation and subsequent polymerization pathway, postulating a passivation model in which layers of immobile unreactive material are formed at the electrode surface inhibiting further reaction. Initially, high molecular weight material is formed which remains in the vicinity of the electrode surface. This leads to a locally rapid molecular weight growth resulting in the differentiation of the polymeric film structure 


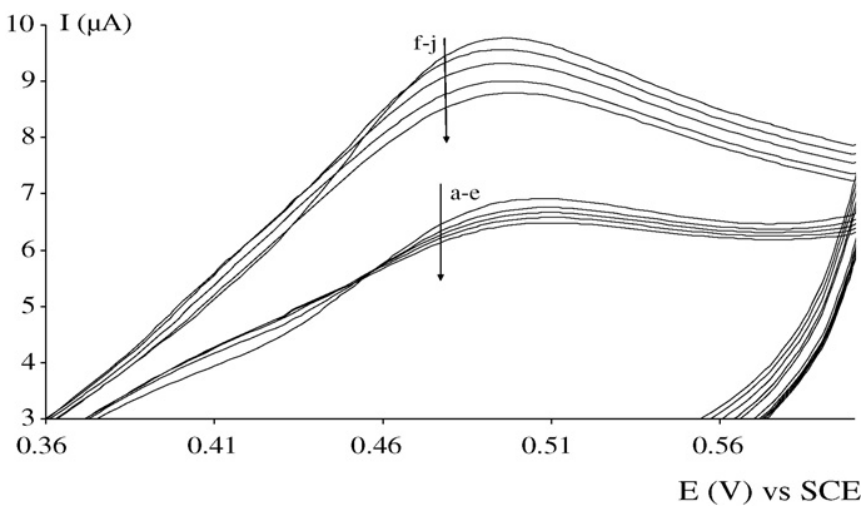

Fig. 3. Current potential curves recorded between 0.6 and $-1.2 \mathrm{~V}$ vs. SCE in a $\mathrm{pH} 12$ buffer solution with a scan rate of $50 \mathrm{mV} \mathrm{s}^{-1}$ at a gold electrode modified with CoTSPc at the breaking point in a $0.6 \mathrm{mmol} \mathrm{L}^{-1} 4-\mathrm{Cp}$ solution with the successive scan numbers (a) 2, (b) 4, (c) 6, (d) 8 and (e) 10 and at a gold electrode modified with CuTSPc during 60 scans in a $0.6 \mathrm{mmol} \mathrm{L}^{-1} 4$-Cp solution with the successive scan numbers (f) 2, (g) 4, (h) 6, (i) 8 and (j) 10 .

in an accelerating manner. Once this immobilized material is oxidized to less reactive forms, the electrode surface becomes effectively shielded for further reaction. Another pathway that can be followed yields quinone-like structures which are water soluble [9]. It is shown that the way in which the phenoxy radical reacts, depends on its formation rate. Higher chlorophenol concentrations and/or low scan rates favour the polymerization and the resulting film behaves as an insulator, passivating the film. On the contrary, lower chlorophenol concentrations and/or high potential scan rates favour the oxidation to quinonic species and the polymer film can be porous enough for charge transfer to continue [9]. Overall, it can be concluded that at low potential scan rates, high chlorophenol concentrations and high $\mathrm{pH}$, which is the case in this study, the formation of films with low porosity is favoured [12].

In this study, it is observed that the net current decreases with $10 \%$ during 10 subsequent voltammetric scans in a $0.6 \mathrm{mmol} \mathrm{L}^{-1} 4-\mathrm{Cp}$ solution for a gold electrode modified with CoTSPc. For the CuTSPc modified gold electrode and the bare gold electrode, the current however decreases respectively with $22 \%$ and $26 \%$ indicating that the fouling of the electrode occurs slower on the gold electrode modified with CoTSPc. An explanation for this difference will be given further in Section 4.

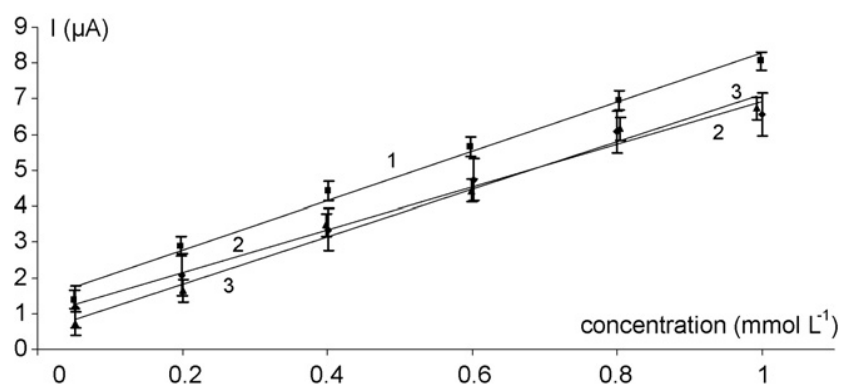

Fig. 4. Calibration curves of 4-Cp oxidation obtained at a bare gold electrode (1), at a gold electrode modified with CoTSPc at the breaking point (2) and at a gold electrode modified with CuTSPc during 100 scans (3) in a pH 12 buffer solution, with a scan rate of $50 \mathrm{mV} \mathrm{s}^{-1}$ at $298 \mathrm{~K}$. The error bars shown are $s_{\mathrm{y}}$.

\subsection{Comparison of calibration curves of $4-\mathrm{Cp}$}

Because of the fouling of the electrode, a fresh electrode is needed for each 4-Cp detection. In addition, only the first cyclic voltammetric scan of the electrode is taken into account to plot a calibration curve. Fig. 4 compares the calibration curves of 4$\mathrm{Cp}$ for a gold electrode modified with CoTSPc till the breaking point (curve 2) and for a gold electrode modified with CuTSPc during 100 voltammetric scans (curve 3). The calibration curve obtained at a bare gold electrode is also shown (curve 1). In Table 1, the slope of the calibration curves $\left(b_{1}\right)$, the intercept with the $Y$-axis $\left(b_{0}\right)$ and their respective uncertainties $\left(s_{b_{0}}\right.$ and $\left.s_{b_{1}}\right)$ are shown together with the pure (experimental) error $\left(\sigma_{\mathrm{y}}\right)$ of the detection of 4-Cp on gold electrodes modified with CoTSPc before, at and after the breaking point. Analogous results for different CuTSPc modified electrodes (17, 60 and 100 voltammetric scans) and for a bare gold electrode are also presented. For CoTSPc, the results show that the uncertainty on the detection of 4-Cp is significantly smaller before the breaking point (CoTSPc), at the breaking point (CoTSPc) and at a bare gold electrode than after the breaking point (CoTSPc). This indicates that the precision decreases with the modification time (increasing voltammetric scan number). Moreover, it is seen that the sensitivity of the 4-Cp detection is significantly lower at the breaking point than before the breaking point and at the bare gold electrode. For the detection of 4-Cp at a gold electrode modified with CuTSPc, it is noticed that the precision and the

Table 1

Overview of the data related to the calibration curves obtained from the electrochemical oxidation of 4-Cp at a bare gold electrode and at gold electrodes electrochemically modified with CoTSPc or CuTSPc in a concentration range of $0.05-1 \mathrm{mmol} \mathrm{L}^{-1}$ with respectively the electrode condition, $b_{1}, s_{b_{1}}, b_{0}, s_{b_{0}}, s_{\mathrm{y}}$ and the number of data points shown

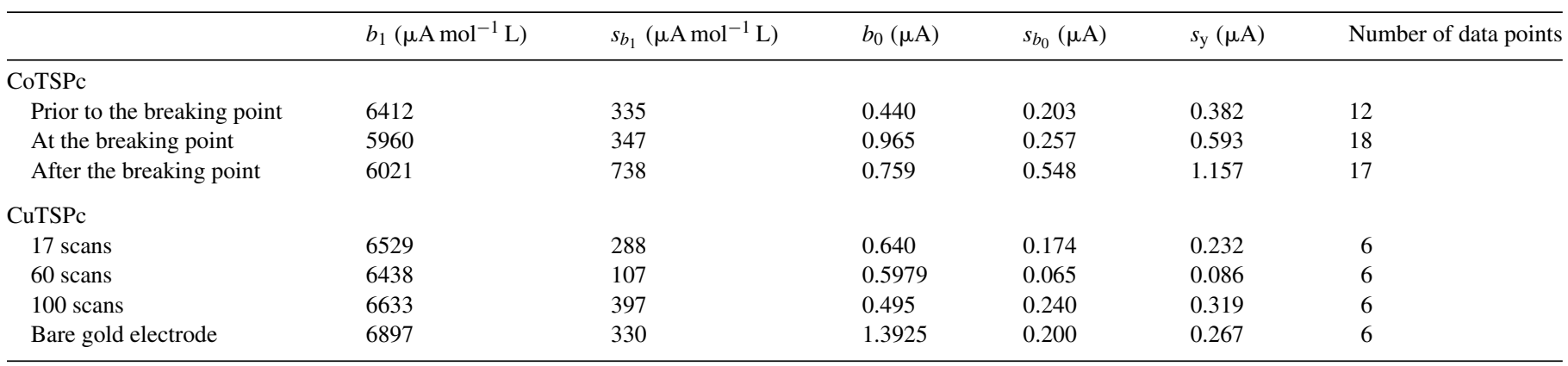


sensitivity are independent from the number of voltammetric scans during the modification process. In contrast to CoTSPc, no significant difference between the detection of 4-Cp at a bare gold electrode and a gold electrode modified with CuTSPc is detected. As can be seen in Fig. 1, the nature of the central metal ion does not affect the oxidation of chlorophenol at the modified electrode. If the latter was true, another cyclic voltammetric behaviour is expected because $\mathrm{Co}(\mathrm{II})$ and $\mathrm{Cu}$ (II) have a different number of d electrons. Based on the above results, it is now clear that the coordination state of the phthalocyanine plays a crucial role in the oxidation of 4-Cp.

\section{Discussion}

The similarity of the detection of 4-Cp at a bare gold electrode and at a gold electrode modified with CuTSPc can be explained by the properties of the phthalocyanine thin film layer. From the first voltammetric scan on, a stable CuTSPc thin film is formed with nicely stacked columnar aggregates. During further modification, the thin film keeps on growing steadily, increasing the number of electrochemically active adsorbed CuTSPc molecules. Because of the four coordinating properties of CuTSPc [17], the phthalocyanine molecules can approach each other very easily. The dense CuTSPc aggregates on the electrode make electron transfer possible through the thin film and have only a minor influence on the electrode sensitivity. This behaviour was observed for all kinds of CuTSPc modified electrodes, explaining the independence of the 4-Cp detection from the number of voltammetric scans during the modification. Moreover, the above results show that the rate of fouling of both the bare gold electrode and the CuTSPc modified electrode is similar, indicating that the oxidation process of $4-\mathrm{Cp}$ at both electrodes is comparable. The modification of the gold electrode with CuT$\mathrm{SPc}$, a four coordinating phthalocyanine, is not an improvement for the detection of 4-Cp.

For CoTSPc, another effect is observed concerning the relationship between the thin film build-up and the detection of 4-Cp. In this case, the change in sensitivity and selectivity for the detection of 4-Cp at a gold electrode modified with CoTSPc before, at and after the breaking point, can also be explained by the change in the thin film build-up during the modification procedure [25-27]. At the start of the modification procedure only electrochemical active monomer species are adsorbed on the gold electrode. On this CoTSPc monomer thin film, monomers as well as dimers are adsorbed. At the breaking point, an equilibrium exists between the monomer and the dimer species. After the breaking point, only adsorption of the electrochemically inactive dimer species on the formed thin film is observed. The similarity in sensitivity for the 4-Cp detection at a gold electrode modified with CoTSPc before the breaking point and at a bare gold electrode can be explained, as for CuTSPc, by the availability of electrochemically active CoTSPc species for the oxidation of 4-Cp. During continuous modification however the number of dimers, adsorbing on the CoTSPc thin film, increases. At the breaking point, the high amount of electrochemically inactive dimer species results in a decrease in the sensitivity of the modified electrode compared to the situation before the break- ing point. During further modification, the amount of dimers on the electrode surface keeps increasing which has a negative influence on the reproducibility of the 4-Cp detection. The presence of the CoTSPc dimers on the surface however appear to have a positive influence on the fouling of the electrode. This can be explained by the more chaotic thin film build-up after the breaking point resulting in a more difficult adsorption of the phenoxy polymer on the surface. It is also possible that the formed phenoxy polymer adsorbs on a CoTSPc dimer site which is not electrochemically active. During this discussion, it is noticed that the build-up of the CuTSPc or CoTSPc thin layers play a crucial role in their electrochemical properties towards the oxidation of 4-Cp. The thin layer build-up is on his turn highly influenced by the coordination properties of the phthalocyanine species used during modification. Four coordinating species, such as CuT$\mathrm{SPc}$, give from the first scan nicely stacked columnar aggregates with electrochemically active monomers and dimers [17]. This has a positive influence on the reproducibility towards the oxidation of 4-Cp in alkaline solution, but there is no improvement of the sensitivity. On the other hand, modification with six coordinating phthalocyanine species, such as CoTSPc, results in a more chaotic layer build-up because of the presence of electrochemically inactive dimer species on the surface [15-17]. The presence of these electrochemically inactive species on the surface has a positive influence on the fouling of the electrode but the reproducibility of the oxidation of 4-Cp decreases markedly which makes this electrode less useful for this oxidation.

\section{Conclusions}

In this paper the oxidation of 4-Cp in a $\mathrm{pH} 12$ buffer solution at a bare gold electrode is compared to its oxidation at a gold electrode modified with CoTSPc or CuTSPc. The modification of the electrode was performed by successive cyclic voltammetric scans in CoTSPc or CuTSPc solutions. It is noticed that the gold electrodes modified with CoTSPc or CuTSPc show no electrocatalytic behaviour concerning the oxidation of 4-Cp. It is, however, seen that the build-up of the CoTSPc or CuTSPc thin film on the gold electrode has a major influence on the sensitivity and the reproducibility of the electrode. High amounts of electrochemically inactive species on the electrode surface have to be avoided. Therefore, four coordinating metal phthalocyanines are preferred and six coordinating metal phthalocyanines have to be avoided as electron transfer mediator. On the other hand, it is noticed that the more chaotic thin film build-up and the presence of electroinactive six coordinating CoTSPc species have a positive effect on the fouling of the electrode surface.

\section{Acknowledgements}

This work was financed by Ghent University (BOF). The authors acknowledge K. Strijckmans (Ghent University) for his help with the statistical calculations.

\section{References}

[1] J. Paasivirta, K. Heinola, T. Humppi, A. Karjalainen, J. Knuutinen, K. Mantykoski, R. Paukku, T. Piilola, K. Surmaaho, J. Tarhanen, L. Welling, 
H. Vihonen, J. Sarkka, Polychlorinated phenols, guaiacols and catechols in environment, Chemosphere 14 (1985) 469-491.

[2] Chlorophenols Other than Pentachlorophenol (Environmental Health Criteria, vol. 93), WHO, Geneva, 1989.

[3] M.T. Galceran, O. Jauregui, Determination of phenols in sea-water by liquid-chromatography with electrochemical detection after enrichment by using solid-phase extraction cartridges and disks, Anal. Chim. Acta 304 (1995) 75-84.

[4] F.E.O. Suliman, S.S. Al-Kindi, S.M.Z. Al-Kindy, H.A.J. Al-Lawati, Analysis of phenols in water by high-performance liquid chromatography using coumarin-6-sulfonyl chloride as a fluorogenic precolumn label, J. Chromatogr. A 1101 (2006) 179-184.

[5] K. Tonokura, T. Nakamura, M. Koshi, Detection of chlorobenzene derivatives using vacuum ultraviolet ionization time-of-flight mass spectrometry, Anal. Sci. 19 (2003) 1109-1113.

[6] U. Stafford, K.A. Gray, P.V. Kamat, Photocatalytic degradation of 4-chlorophenol: the effects of varying $\mathrm{TiO}_{2}$ concentration and light wavelength, J. Catal. 167 (1997) 25-32.

[7] M.O. Azzam, M. Al-Tarazi, Y. Tahboub, Anodic destruction of 4chlorophenol solution, J. Hazard. Mater. B 75 (2000) 99-113.

[8] G. Waldner, M. Pourmodjib, R. Bauer, M. Neumann-Spallart, Photoelectrocatalytic degradation of 4-chlorophenol and oxalic acid on titanium dioxide electrodes, Chemosphere 50 (2003) 989-998.

[9] M.S. Ureta-Zanartu, P. Bustos, C. Berrios, M.C. Diez, M.L. Mora, C Gutierrez, Electrooxidation of 2,4-dichlorophenol and other polychlorinated phenols at a glassy carbon electrode, Electrochim. Acta 47 (2002) 2399-2406.

[10] G.W. Muna, N. Tasheva, G.M. Swain, Electro-oxidation and amperometric detection of chlorinated phenols at boron-doped diamond electrodes: a comparison of microcrystalline and nanocrystalline thin films, Environ. Sci. Technol. 38 (2004) 3674-3682.

[11] M.A. Rodrigo, P.A. Michaud, I. Duo, M. Panizza, G. Cerisola, C. Comnellis, Oxidation of 4-chlorophenol at boron-doped diamond electrode for wastewater treatment, J. Electrochem. Soc. 148 (2001) D60-D64.

[12] M.S. Ureta-Zanartu, P. Bustos, M.C. Diez, M.L. Mora, C. Gutierrez, Electro-oxidation of chlorophenols at a gold electrode, Electrochim. Acta 46 (2001) 2545-2551.

[13] M. Gattrell, B. MacDougall, The anodic electrochemistry of pentachlorophenol, J. Electrochem. Soc. 146 (1999) 3335-3348.

[14] T. Mafatle, T. Nyokong, Use of cobalt(II) phthalocyanine to improve the sensitivity and stability of glassy carbon electrodes for the detection of cresols, chlorophenols and phenol, Anal. Chim. Acta 354 (1997) 307-314.

[15] M.S. Ureta-Zanartu, C. Berrios, J. Pavez, J. Zagal, C. Gutierrez, J.F. Marco, Electrooxidation of 2-chlorophenol on polyNiTSPc-modified glassy carbon electrodes, J. Electroanal. Chem. 553 (2003) 147-156.

[16] K.J. Balkus, M. Eissa, R. Levado, Oxidation of alkanes catalyzed by zeoliteencapsulated perfluorinated ruthenium phthalocyanines, J. Am. Chem. Soc. 117 (1995) 10753-10754.

[17] A. Andreev, V. Ivanova, L. Prahov, I.D. Schopov, Catalytic activity of monomeric and polymeric cobalt(II)phthalocyanines in sulfide ion oxidation, J. Mol. Catal. 95 (1995) 197-201.

[18] K. De Wael, P. Westbroek, E. Temmerman, Electrocatalytic oxidation of dithionite at a cobalt(II)tetrasulfonated phthalocyanine and 5,10,15,20tetrakis-(4-sulfonatophenyl)-porphyrin cobalt(II) modified gold electrode in alkaline solution, Electroanalysis 17 (2005) 263-268.

[19] K. De Wael, P. Westbroek, E. Temmerman, Study of the deposition of a cobalt(II)tetrasulfophthalocyanine layer at gold surfaces in alkaline solution, J. Electroanal. Chem. 567 (2004) 167-173.

[20] Y.-C. Yang, J.R. Ward, R.P. Seiders, Dimerization of cobalt(II) tetrasulfonated phthalocyanine in water and aqueous alcoholic solutions, Inorg. Chem. 24 (1985) 1765-1769.
[21] M. Thamae, T. Nyokong, Interaction of sulfur dioxide and cyanide with cobalt(II) tetrasulfophthalocyanine in aqueous media, Polyhedron 21 (2002) 133-140.

[22] P.J. Camp, A.C. Jones, R.K. Neely, N.M. Speirs, Aggregation of copper(II) tetrasulfonated phthalocyanine in aqueous salt solutions, J. Phys. Chem. A 106 (2002) 10725-10732.

[23] K. De Wael, K. Peeters, D. Bogaert, H. Buschop, L. Vincze, A. Adriaens, Electrochemical and spectroscopic characterization of a gold electrode modified with $3,4^{\prime}, 4^{\prime \prime}, 4^{\prime \prime \prime}$-copper(II) tetrasulphonated phthalocyanine, J. Electroanal. Chem. 603 (2007) 212.

[24] K. De Wael, P. Westbroek, P. Bultinck, D. Depla, P. Vandenabeele, A. Adriaens, E. Temmerman, Study of the deposition and Raman and XPS characterization of a metal ion tetrasulphonated phthalocyanine layer at gold surfaces: density functional theory calculations to model the vibrational spectra, Electrochem. Commun. 7 (2005) 87-96.

[25] K. Peeters, K. De Wael, L. Vincze, A. Adriaens, Comparison of different surface modification techniques for electrodes by means of electrochemistry and micro-synchrotron radiation $\mathrm{X}$-ray fluorescence. Dimerization of cobalt(II) tetrasulfonated phthalocyanine and its influence on the electrodeposit ion on gold surfaces, Anal. Chem. 77 (2005) 5512-5519.

[26] K. Peeters, K. De Wael, A. Adriaens, G. Falkenberg, L. Vincze, Nondestructive characterization of CoTSPc electrochemically deposited on gold electrodes by means of synchrotron X-ray microfluorescence, Electrochem. Commun. 7 (2005) 1157-1162.

[27] K. Peeters, K. De Wael, A. Adriaens, G. Falkenberg, L. Vincze, Quantitative synchrotron micro-XRF study of CoTSPc and CuTSPc thin-films deposited on gold by cyclic voltammetry, J. Anal. Atom. Spectrom. 22 (2007) 493-501.

[28] D.L. Massart, B.G.M. Vandeginste, L.M.C. Buyens, S. De Jong, P.J. Lewi, J. Smeyers-Verbeke, Handbook in Chemometrics and Qualimetrics, Part A, Elsevier, Amsterdam, 1997.

[29] M. Gattrell, D.W. Kirk, A Fourier-transform infrared-spectroscopy study of the passive film produced during aqueous acidic phenol electrooxidation, J. Electrochem. Soc. 139 (1992) 2736-2744.

[30] M. Gattrell, D.W. Kirk, A study of electrode passivation during aqueous phenol electrolysis, J. Electrochem. Soc. 140 (1993) 903-911.

[31] M. Gattrell, D.W. Kirk, A study of the oxidation of phenol at platinum and preoxidized platinum surfaces, J. Electrochem. Soc. 140 (1993) $1534-1540$

\section{Biographies}

Karl Peeters received his $\mathrm{PhD}$ at Ghent University (Belgium) on the topic of sensor development using modified electrodes. He now works for Johnson \& Johnson.

Karolien De Wael works at Ghent University (Department of Analytical Chemistry) as a postdoctoral fellow of the Research Foundation - Flanders. She received her diploma (MSc) and her doctorate $(\mathrm{PhD})$ in chemistry, in 2001 and 2005, respectively, both from Ghent University. Her research interests include the fundamental study of the immobilization of phthalocyanines, porphyrins and enzymes onto electrodes and their characterization.

David Bogaert started his $\mathrm{PhD}$ in the same research group and will continue the work on modified electrodes.

Annemie Adriaens graduated with a PhD in analytical chemistry in 1993 from the University of Antwerp (Belgium), where she was involved in the optimization of new technologies for inorganic micro and trace analysis. In 2001, she became professor in analytical chemistry at Ghent University where she now leads the research group "Electrochemistry and Surface Analysis". 